\title{
Planes de empleo y reforma administrativa
}

Alberto Palomar Olmeda *

\section{Introducción general: el contexto de la reforma}

El análisis que se realiza en el presente trabajo es muy probable que en puridad conceptual, tomando como fundamento el rigor lógico de las actuaciones, debiera comenzar por analizar el contexto general de la reforma administrativa, empezando por determinar la propia necesidad de la misma, para, a continuación abordar sus instrumentos esenciales, entre los cuales debe encontrarse necesariamente la búsqueda de nuevos horizontes en la gestión pública de los recursos humanos muy centrada en el análisis de su propio marco y a menudo muy desconectada de su propia operatividad global y, por tanto, de su conexión con los fines mutantes de las organizaciones en las que se incluyen.

Comencemos por señalar, a este respecto, que la evolución de los fines estatales y especialmente la imbricación de los conceptos Estado-sociedad que, como se ha dicho, es la consecuencia del nuevo modelo de Estado ${ }^{1}$, conlleva necesariamente una transformación del papel de la Administración pública de nuestros días y, por ende, de las formas y técnicas de actuación de la misma, con el objeto de poder cumplir con los nuevos fines estatales.

Este proceso de transformación ha sido objeto de diversos análisis desde los más variados ámbitos doctrinales y administrativos ${ }^{2}$. Aceptemos a modo de punto de partida las palabras de GARCíA DE ENTERRía ${ }^{3}$ quien nos recuerda que en la estructura de Estado inspirada en la clásica división de poderes no tiene cabida una Administración como la de nuestros días, nacida de la Revolución francesa y que postulaba entre sus grandes principios el de reformar la estructura social, para lo cual era necesario un fuerte poder diferente a los señalados en la concepción tripartita y capaz de servir de motor del cambio. Este mismo aspecto es reconocido por PAREJO cuando señala que una de las causas de la transformación administrativa es la "ruptura de la imagen tradicional de identificación de los poderes en sentido de organización con las funciones del Estado... con progresiva formalización de éstas..." ${ }^{\text {. }}$.
Este proceso supone una transmutación del poder ejecutivo que, como indica el propio GarCía DE ENTERRía ${ }^{5}$, no es sólo de cantidad sino, sobre todo, de calidad. A este respecto indica que "el supuesto poder ejecutivo se ha sustantivado en un sujeto real y verdadero, autosuficiente para su desenvolvimiento en virtud de los mecanismos técnicos que ya consideramos, que le aseguran su exención respecto de los otros poderes. Es realmente un sujeto que actúa, persiguiendo como todo sujeto, multitud de fines, no limitados, por supuesto, al simple resultado coactivo de las leyes y de las sentencias".

No es nuestro propósito analizar ahora las diferentes tesis sobre la naturaleza jurídica de la Administración pública, pero sí resaltar que la Administración a la que aquí se hace referencia es algo más que un mero instrumento ejecutor de las normas que dicta otro poder dentro del Estado. Su individualidad propia, su diferenciación mimética de los poderes estatales o de las funciones de éste, su personalidad jurídica, y la pérdida de valor programador que siempre había tenido la Ley, hacen del concepto Administración pública un concepto con entidad sustantiva independiente, puesto que a través de ella se cumplen materialmente los fines del Estado plasmados o no en normas con el rango formal de Ley.

La Administración de nuestros días cumple sus funciones de ejecución de la ley y otras que ella misma establece en virtud de su potestad reglamentaria y organizatoria, a la vez que un conjunto de actividades materiales y de pura ejecución cuya cobertura se encuentra en su propia labor planificadora (plasmada o no en normas jurídicas), o simplemente con la habilitación que le otorga la cobertura presupuestaria ${ }^{6}$.

La cuestión que tradicionalmente se ha suscitado es la de determinar cuáles son los métodos y las formas que se utilizan para ejecutar materialmente este conjunto de actividades. Este debate histórico puede sintetizarse en dos grandes problemas: las formas de la gestión pública y la individualidad de las técnicas de gestión. Por lo que a la primera cuestión se refiere, podriamos, siguiendo la división efectuada por FORSTHOFF ${ }^{7}$, afirmar, en primer término, que la gestión de los intereses y fines del Estado puede hacerse por la propia Administración directamente o a través de lo que dicho autor denominó la "Administración estatal mediata". 
Esta primera división tampoco resulta definitivamente esclarecedora, ya que la denominada Administración directa no es un concepto homogéneo sino que obviamente el mismo está en función del tipo de Estado, pues no es igual la estructura y funcionamiento de la Administración en un Estado unitario, que en un Estado federal o regional ${ }^{8}$.

Indicaba FORSTHOFF ${ }^{9}$ que la característica común a todos los Estados federales es que "realizan la articulación en la esfera constitucional, pero dejan a los Estados miembros su propia Constitución y, con ello, la posibilidad de una formación de la propia voluntad política dentro de los límites dados por la existencia del Estado global". En síntesis y para concluir con la enunciación del problema, debemos recordar las palabras del propio FORSTHOFF ${ }^{10}$ cuando afirma que "toda articulación en la esfera constitucional tiene alguna influencia sobre la Administración".

En los momentos actuales, como señalábamos anteriormente, el debate no puede considerarse centrado en exclusiva en las formas de gestión aunque las cuestiones de mayor calado como la titularidad de los servicios constituya un eje insoslayable del mismo. Sin perjuicio de lo anterior, es lo cierto que existe un conjunto de problemas que conforman una demanda común frente a todas las formas de prestación de servicios y que se centran en las técnicas del funcionamiento del propio servicio. Este tronco común estaría representado por la calidad de los servicios - expresión que utilizamos en este momento en un sentido vulgar-, por la búsqueda de la eficacia gestora y por la eficiencia en la utilización de los recursos, en otras palabras, lo que hoy se conoce como management. A nuestro juicio, y sin menospreciar la discusión sobre el volumen de lo público, es absolutamente consustancial a nuestros días que el debate se centra en la necesidad de prestar los servicios públicos desde parámetros de calidad en la gestión pública que permitan una mayor legitimación de la misma frente a la propia sociedad, de modo que deje de presentarse la Administración de lo público desde posiciones irreconciliables con esa sociedad que a menudo la vive como un mal inevitable del que debe "tirar" sin más justificación, en muchos casos, que la propia inercia histórica de las sociedades de nuestros tiempos.

La asunción de mayores fines y tareas por el Estado, la diversidad de las mismas ${ }^{11}$, su complejidad y naturaleza hacen que la asunción directa de la gestión por los propios órganos administrativos comience a ser cuestionada. Este cuestionamiento constituye, debe constituir un estimulo permanente para la búsqueda de soluciones que aproximen a la sociedad un ámbito gestor del que quizá no pueda disponerse, pero que en todo caso debe afrontar un permanente reto de superación. Reto del que, por otra parte, muy probablemente no pudieran salir victoriosos los rutilantes esquemas privados que, a mi juicio, no han probado su idoneidad para afrontar, seriamente y sin grandes traumas ni rupturas, un proceso del calibre y con los condicionantes de todo orden del que viven nuestras Administraciones Públicas, abocadas a transmutar sus esquemas garantistas y esencialmente reguladores por otros en lo que prima la concepción real de servicio y de gestión de un fuerte acervo prestacional.
Llegados a este punto es forzoso recapitular y señalar a modo de síntesis que el panorama descrito hasta el momento no es otro que el de un modelo de Estado, el denominado Estado social, con unos fines y objetivos que distan de aquellos que los ciudadanos de otras épocas históricas podían demandar de su Estado y que convierten a éste en un elemento activo en la vida social, para asegurar efectivamente la prestación de aquellos servicios.

Lo cierto es que en los últimos tiempos dicho modelo de Estado ha comenzado a ser cuestionado, casualmente coincidiendo con nuevas crisis económicas. En todo caso, este cuestionamiento está directamente relacionado con el volumen y la propia magnitud del crecimiento del sector público en los últimos años, que a principios de los ochenta se situaba en todos los países de la CEE en torno al 50\% del Producto Interior Bruto y que se mantiene en los momentos actuales en torno al 45\%12. Las principales razones del incremento del gasto público están centradas, según ARIÑo, en el peso del denominado gasto social (educación, sanidad y seguridad social), que alcanza entre el $35 \%$ y el $40 \%$ del gasto estata ${ }^{13}$. Este crecimiento y el peso real del mismo en las magnitudes económicas de los países ha sido objeto de algún cuestionamiento al hilo, como señala ARIÑo, de que " los economistas subrayan que la demanda de cualquier bien normal a precio cero tiende a ser infinita, por lo que si no se produce un racionamiento vía precios... se produce un deterioro de la calidad y un racionamiento vía colas y lista de espera..." ${ }^{14}$. Este cuestionamiento, ideologías aparte, no va a apartarnos del camino inicial. A nuestro juicio, lo que puede y debe ponerse en cuestión es el volumen y la situación estratégica del Estado en la vida social pero no su presencia, ya que resulta evidente que el relanzamiento industrial, la actividad económica $y$, en general, lo que ahora se denomina vulgarmente el mercado, han demostrado suficientemente en la historia su imposibilidad de actuar como verdaderos mecanismos reequilibradores de las necesidades sociales. Y que, finalmente, sin ese efecto de solidaridad y equilibrio es muy difícil mantener la paz social en nuestros días. Por tanto, a nuestro juicio, es perfectamente lícito y razonable el debate del monto global de la presencia estatal y aun referido a su presencia sectorial, pero no el de la necesidad de que el Estado actúe como elemento de equilibrio y solidaridad social, que debe estar fuera de toda duda.

En todo caso y centrada así la cuestión, lo verdaderamente relevante es el análisis de cómo las Administraciones Públicas contribuyen a hacer efectivo este proceso, máxime en épocas como la presente en la que la economía y el coste de las medidas puede condicionar la propia existencia o implantación de éstas. En esta línea no es aventurado indicar que la Administración pública, en cuanto conjunto de elementos orgánicos, personales y materiales, no es ajena a dicha transformación, sino que, muy al contrario, debe buscar fórmulas para responder adecuadamente a las demandas sociales que se le impongan. La más sencilla de las fórmulas de gestión, que se representaría en la asunción por el propio aparato administrativo de las nuevas funciones a desarrollar, se presenta como una fórmula que condiciona su propio futuro, ya que, en la misma medida que se pro- 
duzca el crecimiento del aparato administrativo, se reduce paulatinamente la eficacia de la gestión al crecer la descoordinación, el solapamiento de funciones, la burocratización -en el sentido peyorativo de la palabra-, etc.

Este planteamiento nos exige insistir, de nuevo, en que la sociedad de nuestros días demanda del Estado cuestiones, prestaciones y aún comportamientos difícilmente encuadrables en los esquemas tradicionales que configuraban la Administración como un mecanismo de ejecución de los mandatos del Parlamento en cuanto representante de los intereses populares.

Sin que, obviamente, pueda postularse el abandono de este esquema sí debemos indicar que el mismo no cubre ni puede cubrir la totalidad de la actuación de la Administración que, en cuanto poder público, debe contribuir a la realización del modelo de Estado. Este papel constitucional de las Administraciones de nuestros días exige conectar el aparato instrumental con un Estado que interviene en la vida social y económica, que se convierte en prestador de ciertos servicios y en garante de otros.

Este esquema superador de las concepciones del Estado policial, que se ha plasmado prácticamente en la totalidad de los textos constitucionales de los países de nuestro entorno, nos presenta un modelo plural de Administración que debe ofrecer a los ciudadanos, por un lado, un cúmulo de prestaciones públicas, ordenando además los sectores más importantes de la vida económica y, por otro lado, la garantía del libre ejercicio de sus derechos fundamentales y libertades públicas.

Este marco global ha producido en las sociedades de nuestro entorno (EE.UU.; Italia; G.B/R.U.) un movimiento de diversa composición (interna, externa, académica, doctrinal) que intenta afrontar un amplio proceso de reforma administrativa para cumplir esos nuevos fines. En resumen, la reforma como un mecanismo de respuesta en los fines y en las formas de satisfacción pública de las demandas sociales.

La reforma es, a menudo, un propósito recurrente en el que con tintes serios y fundados, en unas ocasiones, o con simples aproximaciones, en muchos casos demagógicas y poco reflexivas respecto de los intereses de la propia organización y, por ende, de la sociedad, se formulan posiciones de cambio de forma casi mecánica. La necesidad de la reforma es en los momentos actuales algo que transciende, incluso, a las formulaciones políticas, ( no así claro está, las formas de realizar la misma), ya que deriva de causas estructurales que no se corresponden con elementos del análisis parcial sino que son consecuencia de factores socialmente constatables y que operan, a nuestro juicio, como elementos de partida ineludibles e indisponibles en cualquier análisis.

Esa necesidad de cambio de formas, de estructuras, de cultu$\mathrm{ra}$, de procedimientos y de opciones exige la imbricación de todos los elementos que configuran su propia esencia. En el presente trabajo se trata de analizar uno de ellos, el de los recursos humanos, que juega un papel relevante desde cualquier óptica a la que queramos aproximar nuestro análisis, tanto desde una perspectiva económica como de servicio, ya que difícilmente puede producirse un cambio si los actores materiales del mismo no son adiestrados para el mismo y si , en suma, no están dispuestos a involucrarse en él.

La Administración Pública de nuestros días debe ser capaz de generar un nuevo clima para los empleados públicos que les sensibilice con las demandas de los ciudadanos, lo cual redundará, sin duda, en una mejora de los servicios, pero también en una mejor sensación de los propios empleados públicos que estarán dispuestos, si se sienten útiles, a defender su importancia para la sociedad. Este no es desde luego un proceso fácil ni que pueda conseguirse en un período de tiempo relativamente pequeño, pero, junto a esto, sí es evidente que se trata de un proceso más fácil de conseguir cuanto antes se comience y, sobre todo, cuanto más se sustituya la retórica por la acción.

\section{Los recursos humanos en el contexto de la reforma administrativa}

En el marco global de reforma que se enunciaba en el apartado anterior nos interesa analizar, en este momento, uno de los aspectos más relevantes de cualquier proceso de reforma organizativa: el de los recursos humanos, cuya importancia es absolutamente relevante como motor y artífice del propio proceso.

En este sentido, es necesario indicar que la Administración Pública de nuestros días está sometida a una serie de procesos transformativos de signo no homogéneo que la enfrentan simultáneamente con el reto de dejar de ser un ente esencialmente regulador para convertirse en una Administración, básicamente, prestadora de servicios. Este proceso se une con otro de alcance cuantitativo, imprescindible para dar satisfacción real a la necesidad de articular un Estado descentralizado territorialmente. En último término y en sentido inverso, es necesario recordar que este proceso debe conjugarse con otro, el procedente de la integración en una organización supraestatal.

Se trata, en suma, de un amplio proceso de transformación realizado de forma simultánea y en un período de tiempo notablemente reducido. La necesidad de que la solución de dicho proceso se realice realmente sin incrementar los costes ni el volumen de gasto en esta materia, y de una forma eficaz para la satisfacción de los intereses respectivos exige que se conjuguen elementos de planificación de carácter general y sobre perfiles macroestructurales, con los puntuales y microestructurales que adapten los primeros a los ámbitos respectivos. Sólo la adecuada conexión de ambos aspectos puede proporcionar coherencia a un proceso que en su plasmación en el ámbito de los recursos humanos podría llegar a ser contradictorio.

No cabe duda de que nos encontramos ante un proceso de gran dificultad, pero es evidente que ésta no puede operar como una excusa indefinida para la operatividad, ya que la demora en el proceso condiciona su propia realización en sus términos actuales y, por ende, del papel de lo público y de la propia 
capacidad de éste para dar satisfacción a las nuevas demandas sociales.

La importancia del proceso que analizamos exige, además, tener presente que dos son los elementos claves en la consideración de los recursos humanos en la actualidad. Por un lado, su propia repercusión en el volumen de los recursos públicos ${ }^{15}$. Su importancia cuantitativa en el gasto público constituye una referencia ineludible en cualquier análisis global del papel de lo público en nuestras sociedades. En segundo termino, y como consecuencia o independientemente de lo anterior, la necesidad de optimizar los recursos humanos disponibles en el seno de cualquier organización constituye un reto gestor innegable del que ya no pueden escapar por más tiempo las Administraciones Públicas, llamadas por su propia realidad a reubicar sus recursos, a no disponer de excedentes innecesarios y, en suma, a adaptar sus recursos a las necesidades cambiantes de las organizaciones públicas ${ }^{16}$.

El conjunto de cuestiones planteadas ha hecho necesario dotarse de elementos e instrumentos que son concebidos en otras áreas de dirección y que, superando visiones microestructurales y esencialmente estáticas, permitan una realización concordada y general de los problemas apuntados. En este sentido, resulta imprescindible instaurar un sistema de planificación estratégico de recursos humanos con el que hacer frente ordenadamente a la subvención de las necesidades sociales, sin recurrir a fórmulas incrementalistas de las que hay que cuestionarse su validez final.

La planificación de los recursos humanos, como en general toda la actividad de programación, es una actividad bastante poco desarrollada en las Administraciones Públicas de nuestros días. Las razones de esta infrautilización responden a un cúmulo de circunstancias que van desde las puramente históricas de tratarse de una función atribuida a la ley, hasta las puramente teóricas que parten de considerar que se trata de una técnica inaplicable en las Administraciones Públicas.

En los mómentos actuales puede afirmarse que la capacidad de programación desde las normas legales dista mucho de ser la que necesita una organización del volumen de la Administración y que, por tanto, la recepción e introducción, con perfiles propios, de las técnicas de dirección científica resulta un fenómeno imparable en tanto supone una clara opción por la gestión profesionalizada que, lejos de ser un modernismo caprichoso, es una exigencia para la realización eficaz de las funciones atribuidas. El reto de la eficacia que tanto ha tensionado nuestras Administraciones Públicas y que tantos debates ha generado en la determinación de los límites y los medios para conseguirlo es, sin duda, un elemento esencial en la configuración constitucional de la Administración Pública y obliga a ésta a adoptar los instrumentos y técnicas necesarios para conseguir aquella. Estamos, pues, ante la necesidad de articular una exigencia constitucional ${ }^{17}$.

No obstante esta actitud general de cambio de instrumentación técnica, es lo cierto que la misma es especialmente sensible en determinados sectores, como el de los recursos humanos, en el que la introducción de instrumentos no convencionales en la dirección pública debe servir para dimensionar adecuadamente las organizaciones y adaptar dichas dimensiones a los fines concretos que, en cada momento desarrolle la misma.

En este sentido, uno de los elementos esenciales que habrá de conectar la planificación que aquí se introduzca debe ser la de configurarla como un instrumento de dirección ligado esencialmente a los fines de la propia Administración, ya que, muy a menudo, las disfuncionalidades se deben precisamente a la falta de coordinación entre los recursos humanos y la organización, de forma que, habiendo cambiado sustancialmente el conjunto de actividades que desarrolla un órgano, o la forma y duración de las mismas, estas variaciones no tienen, sin embargo, traducción real en el volumen de personal del mismo. La necesidad de optimizar los recursos disponibles con una política de mayor eficiencia en la utilización de los recursos humanos se demuestra esencialmente en circunstancias como las que señalábamos.

En el ámbito que estamos analizando podemos comenzar por indicar que no existe en nuestro país una tradición de planificación general de los recursos humanos, cuyos incrementos pequeños o notables, o sus reducciones/disminuciones no son el futuro de una reflexión sobre el papel que se atribuye a la respectiva Administración Pública, sino que, en la mayor parte de las ocasiones, las justificaciones últimas de aquellas responden a factores económicos generales y más concretamente a la determinación de si nos encontramos en épocas de bonanza o de crisis económica.

Esta insuficiencia en los planteamientos se presenta como un elemento importante de distorsión y de indisciplina ya que permite, en las épocas de crecimiento, obtener y solucionar los problemas de gestión acudiendo al factor recursos humanos como determinante de una opción gestora, que en los momentos de crisis no pueden ser captados con idéntica facilidad y que acaban condicionando la propia opción gestora. No es éste el momento para recalcar, aunque sí conviene recordarlo, que el reclutamiento de recursos debe responder a las auténticas necesidades de la organización y que éstas responden a una lógica directamente ligada a la organización, lo que debe excluir la utilización de aquel proceso con fines políticos y como elemento de reactivación social en momentos de depresión económica. Lo que sí es cierto y no cabe negarlo, es que para eliminar o reconducir las tensiones a los aspectos puramente organizacionales es necesario que la organización esté dotada de los elementos esenciales para responder afirmativa o negativamente sobre la racionalidad de cualquier demanda de recursos humanos. La insuficiencia de la planificación macroestructural responde a las propias carencias de la programación general, pero, en el presente supuesto, es también reflejo del propio carácter rudimentario de los elementos de programación específica que no siempre permiten formular adecuadamente aquel criterio.

En este sentido, no es vano indicar que nuestras normas de función pública han estado tradicionalmente ajenas a los fenómenos de planificación de los recursos humanos, entre otras consideraciones porque, hasta la publicación de la Ley 30/1984, de 2 de Agosto, de Medidas para la Reforma de la Función 
Pública, la gestión del personal y ,por tanto, todas las labores de programación y ejecución se centraban en el instrumento "cuerpo" de funcionarios que, por su desconexión con la propia estructura y organización, era un mecanismo esencialmente inválido para fundar en él cualquier pretensión de planificación global de las necesidades de recursos humanos.

Con la introducción en la Ley de 1984 del concepto "Relaciones de Puestos de Trabajo" puede decirse que se produce un primer paso para cambiar las cosas, dado que, a partir de ese momento, es posible enfrentar a los órganos administrativos con sus necesidades en materia de recursos humanos. Sin embargo, el resultado de este proceso no es enteramente satisfactorio, ya que las relaciones de puestos de trabajo proporcionan una visión estática, no dinámica, de las organizaciones, de forma que la introducción de elementos no estáticos exige necesariamente cambiar la propia relación de puestos lo cual, en términos generales, impide realizar una verdadera labor planificadora y, por ende, impide poner en práctica medidas como la redistribución de efectivos, entre otras, esenciales en el seno de una Administración Pública como la actual, sometida a un proceso de transformación del calado del que se da en nuestros días.

A este propósito global y dinámico sobre el que poder fundar otros juicios más generales, responde la introducción en la Ley 22/1993, de 26 de diciembre, de Medidas Fiscales de Régimen Jurídico de la Función Pública y de la Protección por Desempleo, del instrumento denominado "plan de empleo", cuyo análisis se realiza a continuación y que constituye, sin duda, un primer elemento determinante de una nueva posición en la forma de gestionar los recursos humanos en el seno de nuestras Administraciones Públicas.

\section{El instrumento "planes de empleo": Aproximación conceptual}

El plan de empleo se configura como un instrumento conjunto e integrado de aproximación a la problemática de los recursos humanos de órganos y unidades administrativas en orden a especificar las necesidades de la organización en un plazo temporal determinado y teniendo en cuenta el rol que dicha organización debe jugar en sus aspectos competenciales durante ese período de tiempo.

El Real Decreto 364/1995, por el que se aprueba el Reglamento General de Ingreso del personal al servicio de la Administración General del Estado y de Provisión de Puestos de Trabajo y Promoción Profesional de los Funcionarios Civiles de la Administración General del Estado, determina una diferenciación conceptual de los planes de empleo en dos categorías: los planes de empleo integrales y los planes de empleo operativos.

Según el artículo 2, son Planes Integrales aquellos que especifican los objetivos a conseguir en materia de personal, los efec- tivos y la estructura de recursos humanos que se consideren adecuados para cumplir tales objetivos, las medidas a implementar para transformar la dotación inicial en la que resulte acorde con la estructura de personal que se pretenda y las actuaciones necesarias al efecto, especialmente en materia de movilidad, formación y promoción. Por su parte, los planes operativos son aquellos que tienen por objeto lograr una mejor utilización de los recursos humanos y a tal efecto determinan las previsiones y medidas a adoptar sobre movilidad, redistribución de efectivos y asignación de puestos de trabajo.

En consecuencia, nos encontramos ante dos instrumentos diferenciados: por un lado, aquel que enfrenta a las diversas organizaciones con su reflexión estratégica global y, por otro lado, aquellos otros que tienden a solucionar problemas puntuales de cualquier organización como la Administración Pública, en la que no pueden utilizarse los expedientes de regulación de empleo ni la salida de los excedentes, que es la técnica que suele utilizarse desde el ámbito privado para solucionar estos problemas.

Esta diferenciación reviste notable importancia, ya que permite redefinir una figura como la de los planes de empleo, que por su falta de precedentes en nuestro Ordenamiento, por el cambio cultural que puede suponer, por el propio instrumento legislativo de establecimiento, que, puede no ser más adecuado, como por las primeras impresiones que produjo en el seno de la Administración, pueden haberse malinterpretado o malentendido ya que se asociaron ineludiblemente (por el conjunto de factores señalados y por las propias medidas que les acompañan) a una concepción traumática, pero que, en modo alguno, ha de considerarse como el propósito esencial de la figura.

Por este motivo, es necesario insistir, de nuevo, en que su finalidad esencial es la de introducir la reflexión estratégica en el seno de las Administraciones Públicas en materia de recursos humanos. El propósito es, en sí mismo, loable y esencialmente legitimador de todo un conjunto de políticas - entre las que debe destacarse la del reclutamiento externo - que, de otro modo, responden a criterios de organización difícilmente aceptables en un contexto de dirección científica. Pero, desde una consideración menos pretenciosa, sí ha de señalarse que su consideración como instrumento global de recursos humanos y, por tanto, como instrumento que, con carácter general, aborda la problemática del personal al servicio de una organización sin diferenciar, a estos efectos, el régimen jurídico al que estén sometidos, debe considerarse un claro avance superador de las disfuncionalidades que se han producido en el seno de las Administraciones Públicas al intentar solucionar un problema que se arrastra desde 1964 y que se identifica con la supervivencia de dos regímenes jurídicos diferenciados en el seno de las Administraciones Públicas: los funcionarios públicos y el personal laboral

Las reflexiones anteriores nos permiten ubicar correctamente un instrumento que de otra forma puede plantear numerosos problemas, no sólo como instrumento de dirección, sino, incluso, de inserción en el marco constitucional, cuestión ésta que ya 
hemos analizado en otra ocasión y a la que debemos remitirnos en este momento ${ }^{18}$.

En este contexto es necesario indicar ahora que el proceso de reflexión en una y otra clase de planes tiene algunos aspectos de identidad esencial y otros marcadamente diferentes, especialmente en lo que se refiere a las medidas finales. Por este motivo, parece oportuno que el análisis que aquí pretendemos pase por enfrentarnos primero con el proceso de reflexión común a ambos y que, a continuación, examinemos las medidas de ejecución donde se concretarán las diferencias.

\section{A) Procedimiento para la planificación estratégica pública}

A nuestro juicio, el proceso de planificación estratégica en el ámbito de los recursos humanos, que se plasma en los Planes de Empleo tiene, conceptualmente hablando, tres grandes aspectos. En primer término, la demostración de la existencia de necesidades en materia de recursos humanos en el seno de una organización, en segundo término, la validación y objetivación de las mismas y, en tercer término, la determinación de un conjunto de medidas de entre las legalmente previstas, para solucionar los problemas y disfuncionalidades apuntadas. Examinemos por separado cada una de estas fases.

\section{a) Diagnóstico sobre las necesidades}

En lo que se refiere a la primera de las cuestiones, es claro que la reflexión estratégica que se propone ha de tener como premisa el análisis del esquema organizativo del órgano en cuestión, de los procesos de trabajo y de las propias cargas de trabajo, de la tecnificación de su funcionamiento y del acervo competencial en el que debe desarrollar su función. Este primer aspecto debe permitirnos un conocimiento de la organización, de su operatividad y funcionamiento. Tal juicio inicial debe conjuntarse con los medios humanos necesarios para realizarlo. Así, este análisis permitiría conocer de qué manera se produce, en el momento del examen, la realización de las propias tareas y en concreto, si dicho proceso es correcto o si, por el contrario, se están utilizando mecanismos de personal de carácter precario o notablemente desviados de lo que podríamos denominar la normalidad de la gestión de los recursos humanos. Esta primera etapa debe servir para determinar, a priori, si nos encontramos ante una unidad administrativa con problemas de desfase cuantitativo -tanto por su consideración de deficitario como por su consideración de excedentario- de recursos humanos, si tiene algún tipo de disfuncionalidad cualitativa -fundamentalmente las que se refieren al régimen jurídico del personal-, o si nos encontramos ante una organización bien dimensionada que generará, por tanto, una serie de previsiones de cobertura de las bajas vegetativas o fenómenos similares que permitan seguir manteniendo la adecuada proporción entre los fines y los medios humanos para la satisfacción de aquellos. Esta fase debe concluir con una propuesta razonada sobre los mecanismos y formas con los que arreglar las necesidades o disfunciones detectadas.

Una novedad importante que merece ser destacada es la relativa a la iniciativa para la planificación estratégica que, según la Ley 22/1993, corresponde al Ministerio u Organismo afectado o, conjuntamente, a los Ministerios para las Administraciones Públicas y de Economía y Hacienda. Es claro, por tanto, que la iniciativa es concurrente, ya que, por un lado, se atribuye al propio órgano afectado y, por otro, y sin ninguna limitación, a los dos Departamentos Ministeriales de carácter y funciones "horizontales" a quienes se les asigna así aquí una labor de vigilancia de la coherencia final del sistema que resulta francamente novedosa.

\section{b) Validación y comprobación de la existencia de necesi- dades}

El segundo paso en la reflexión estratégica debe venir representado por la validación o comprobación de la reflexión operada y de los términos concretos de la misma. Así como el protagonismo de la primera fase debe corresponder esencialmente al organismo gestor, en esta segunda fase el protagonismo debe corresponder a los Departamentos que desarrollan funciones de carácter horizontal en el seno de las Administraciones Públicas de nuestro entorno, precisamente porque son ellos los llamados a asumir las consecuencias que, en el plano general, se derivan del diagnóstico estratégico realizado en la fase anterior. No es momento de detallar las formas de realización de la validación, pero sí debemos indicar en este momento que la misma debe alcanzar, en primer término, a la determinación de las cargas de trabajo y, en general, a la de que el juicio de organización y funcionamiento en el que se funda el propio plan es correcto y no enmascara otros problemas de organización y funcionamiento que tan habituales son en nuestros días, y frente a los cuales se suele formular como pretensión esencial la de incrementar los recursos humanos cuando dichas necesidades pueden ser subvenidas por otros procedimientos gestores u organizativos. En segundo término, esta es una fase que debe servir para contrastar la bondad de las medidas propuestas y su idoneidad para ser instrumento esencial en la solución de los problemas planteados.

La realización de la verificación técnica de las necesidades y de las soluciones propuestas produce un claro fenómeno de objetivación de la problemática específica que permite afrontar, sin dilaciones, la tercera de las fases: negociación y aprobación.

\section{c) Negociación y aprobación del Plan de Empleo}

Esta tercera fase tiene por objeto último aprobar formalmente el plan de empleo. No obstante, es necesario indicar que, de conformidad con lo establecido en la Disposición Adicional Séptima de la Ley 22/1993, los planes de empleo deben ser objeto de negociación con las Organizaciones Sindicales más representativas en los términos que establece la Ley 9/1987, de 12 de junio, de Organos de Representación, Determinación de las condiciones de Trabajo y Participación del Personal al Servicio de las Administraciones Públicas. Esta negociación debería versar, a nuestro juicio, sobre el conjunto de medidas a aplicar para solucionar unas necesidades que, a estas alturas de la reflexión, deberían estar plenamente acreditadas y respecto de las cuales no debería abrirse nuevo debate. A este proceso de objetivación 
contribuiría el que la negociación centralizada que, por imperativo legal, se impone en este momento, fuese acompañada en las fases anteriores de la participación y colaboración de los representantes sindicales y demás órganos de representación funcionarial y laboral.

Este doble proceso permitiría escindir la reflexión en dos etapas formalmente diferenciadas: primera, que tendría por objeto determinar la existencia de necesidades, proceso al que pueden contribuir de forma muy eficaz los representantes de los empleados públicos cuyo ámbito de representación se corresponda estrictamente con el analizado; $y$, la segunda, en la que, una vez claro y validado el diagnóstico, se efectúa una negociación sobre el conjunto de medidas con las que va a solucionarse el problema planteado, fase para la cual la representación centralizada adquiere una mayor consistencia, en razón a la necesaria homogeneidad que deben tener en su aplicación y a la necesidad de presentar este proceso como un proceso conjunto en el que una larga serie de medidas aparecen encadenadas.

Esta fase concluye con la aprobación del Plan de Empleo que según el art. 2.3. del Reglamento de Ingreso corresponde al Secretario de Estado para la Administración Pública, previo informe del Ministerio de Economía y Hacienda. Este informe que se concibe, desde la ley, en el marco de las más clásicas facultades de control que, tradicionalmente, han correspondido al citado Departamento, resultara un mero trámite testimonial si, como es de esperar, los Departamentos ministeriales con competencias en el plano horizontal realizan todo este proceso de consuno.

\section{B) Las medidas de ejecución de los planes de empleo}

Como anteriormente se ha dicho, uno de los principales problemas que han presentado los planes de empleo es el de la identificación del instrumento con el conjunto de medidas que en él se podían prever, según la propia Ley 22/1993, y que, en una primera aproximación, hizo que fueran concebidos como una medida traumática semejante a la que en el ámbito laboral suponen los expedientes de regulación de empleo. Esta visión significa, a los efectos de lo que aquí se analiza, confundir la parte con el todo, ya que el hecho de que potencialmente pueda existir un juicio de reflexión de carácter excedentario que exija procesos de reasignación, no puede llevarnos a confundir un panorama que tiene mucha mayor amplitud y que intenta aproximar a las Administraciones Públicas a un marco de dirección científico, no improvisada ni arbitraria, que la haga funcionar como una organización estable y no al albur de los impulsos políticos, cuya influencia, organizativamente hablando, es necesario reconducir ${ }^{19}$.

Desde nuestra consideración y en la línea de lo apuntado, es necesario deshacer un primer equívoco y señalar que las medidas de ejecución de un plan de empleo no son en exclusiva las que se señalan en la Ley 22/1993; éstas son algunas de las posibles, pero en modo alguno las únicas, ya que el plan de empleo, como instrumento de planificación global, entronca con el Ordenamiento de la Función Pública considerado en su conjunto y, por tanto, resultan aplicables la totalidad de medidas de gestión que sean necesarias para llevar a cabo la ejecución de la planificación apuntada. Es más, si se tiene en cuenta que la reflexión que suponen los planes de empleo alcanza también al personal laboral, debería incluirse el conjunto de medidas y procedimientos que permiten conseguir en el ámbito laboral los efectos previstos en un plan de empleo y, entre ellos, los encaminados a disciplinar u ordenar la movilidad ${ }^{20}$.

Sin perjuicio de lo anterior, parece conveniente, dada su novedad y su afección directa a conseguir mayores cotas de gestión en la administración de personal, que analicemos ahora, siquiera sea someramente, las medidas más importantes que para conseguir la ejecución de la planificación estratégica se introducen en nuestro Ordenamiento Jurídico en la Ley 22/1993.

\section{- Relativas a la movilidad.}

Se incluye en este apartado la prescripción relativa a la suspensión de la incorporación de personal externo, tanto el procedente de la Oferta de Empleo como de los procesos de movilidad general.

En una organización donde difícilmente puede operarse sobre perfiles personales, tanto por razones legales como por la propia realidad de la cosas, una medida como la propuesta debe hacerse compatible con el principio de mérito y capacidad y, por ende, con el de objetividad, para evitar que a los puestos no llegue el "despojo" de la organizaciones, sino aquellos a quienes les corresponda en el marco de procesos generales.

En segundo término, podemos referirmos a la reasignación de efectivos de personal que técnicamente se articula en la Ley 30/1984, en cuyo artículo 20.1 (nuevo apartado, el g), LMFRFP y PD), con el fin de regular la reasignación de efectivos como consecuencia de la supresión de puestos de trabajo a resultas, a su vez, de un plan de empleo. Este proceso se configura en la Ley 22/1993 sobre la base de tres fases, en las que se compaginan el protagonismo del ente gestor con el Ministerio para las Administraciones Públicas y del Ministerio de Economía y Hacienda.

Como criterio general y antes de efectuar el análisis de su regulación concreta en las diferentes fases, es necesario indicar que el nuevo apartado g) intenta objetivar el proceso con una declaración de alcance general, según la cual "la reasignación de efectivos como consecuencia de un Plan de empleo se efectuará aplicando, criterios objetivos relacionados con las aptitudes, formación, experiencia y antigüedad que se concretarán en el mismo".

\section{- Medidas de redimensionamiento incentivado.}

Nos referimos, en primer término, al artículo 24 LMFRFP y PP que, adiciona un nuevo apartado 7 al artículo 29 de la Ley 30/1984, de 2 de agosto, de Medidas para la Reforma de la Función Pública.

Con carácter general, podemos indicar que dicho apartado tiene por objeto crear la situación de excedencia voluntaria incentivada. A ella pueden aspirar únicamente los funcionarios que se encuentren en cualquiera de las dos primeras fases del 
proceso de reasignación de efectivos, o quienes se encuentren en expectativa de destino o excedencia forzosa por aplicación de un plan de empleo.

Su regulación podemos resumirla en torno a los siguientes parámetros:

- El incentivo es una mensualidad de las retribuciones de carácter periódico -excluidas las pagas extraordinarias y el complemento de productividad-, devengados en el último puesto de trabajo desempeñado por cada año de servicio efectivo y con un máximo de doce mensualidades.

- La baja que es temporal, ya que se indica que en esta situación se permanece durante cinco años, en los que no se puede trabajar en ningún puesto público.

En segundo término, debemos analizar en este apartado el artículo 27 LMFRFP y PD que adiciona un nuevo artículo 34 a la Ley 30/1984, de 2 de agosto. La medida propuesta puede resumirse, fundamentalmente, en la idea de que con la misma trata de establecerse un modelo de jubilación anticipada incentivada.

Se configura como una medida aplicable únicamente a quienes estén afectados por un Plan de Empleo, estén en expectativa de destino $o$ en excedencia forzosa y tengan, al menos, 60 años de edad.

- El incentivo es una indemnización, por una sola vez, de una cantidad que fijará el Gobierno según la edad y las retribuciones íntegras correspondientes a la ultima mensualidad completa devengada, con exclusión, en su caso, del complemento específico y de la productividad, referida a doce mensualidades.

- Las condiciones de la jubilación anticipada son las que, respectivamente, se establezcan en el correspondiente régimen de seguridad social, lo cual significa que resultan aplicables los porcentajes de reducción por edad en el régimen general de la seguridad social, además de referir el cálculo a la base reguladora calculada a los sesenta años, etc...

\section{- Medidas específicas de promoción interna.}

Las especiales características de la promoción interna y su inclusión indudable en el ámbito de la reserva de ley a la que se refiere el artículo 103.3 de la Constitución hace que las prescripciones que legítimamente podrían incluirse en el ámbito de un plan de empleo no sean innovadoras sino exclusivamente aplicativas en lo concreto y para un ámbito determinado de las reglas generales de la promoción interna.

En todo caso y en la línea de objetivación que se propone como imprescindible para compatibilizar este instrumento con el marco general derivado de la determinada ley debe reseñarse las que derivan del Acuerdo Administración-Sindicatos de 15 de septiembre de 1994, que puede operar como elemento interpretativo y aclaratorio del alcance de la medida propuesta.

Es necesario indicar que la misma concuerda, aunque no con carácter excluyente, con la del establecimiento de cursos de formación y capacitación que, evidentemente, tendrán mucho que ver en realización de las medidas específicas de promoción interna.

\section{- Medidas relativas a la jornada de trabajo.}

En este apartado se incluye, básicamente, la medida relativa a la prestación de servicios a tiempo parcial. Es esta una medida cuya implantación real tanto en el ámbito funcionarial como en el laboral, presenta notables dificultades especialmente si su utilización pretende hacerse con carácter retroactivo y no meramente innovativo de cara a las futuras incorporaciones.

No obstante sus dificultades prácticas, es lo cierto que se trata de una medida con la que pueden solucionarse algunos de los problemas reales existentes en nuestras organizaciones públicas, especialmente, en el ámbito local.

\section{A modo de conclusión}

No es un tópico indicar a estas alturas la necesidad de una reforma administrativa. Las causas que justifican una conclusión de este orden subsisten 0 , incluso, se han visto incrementadas como consecuencia de un proceso de transformación finalista del Estado que necesita dotarse de nuevos elementos gestores, de nuevas estructuras y pautas de comportamiento con el que satisfacer las nuevas demandas. Por el contrario, sí podría considerarse un tópico aquella referencia si no se aportan elementos tangibles y diferenciales con los que conseguir la deseada reforma.

A nuestro juicio, éste debe ser el camino, la Administración Pública de nuestros días, sometida a un proceso transformador como el apuntado, debe ser capaz de dotarse de elementos técnicos que permitan unas pautas de comportamiento diferentes $y$, en todo caso, más satisfactorias para los intereses sociales.

Este es el marco en el que debe ubicarse conceptual y operativamente el instrumento denominado plan de empleo. El propósito, como ya hemos indicado, es la introducción en el ámbito de las Administraciones Públicas de la reflexión estratégica en un funcionamiento son habituales en el seno de todas las organizaciones minimamente habituadas con técnicas de gestión cientificamente validadas, pero que han estado tradicionalmente ausentes de la gestión pública cuyo nivel de permeabilidad a aquellas ha sido notablemente reducido.

El reto de la eficacia y de la eficiencia al que ya no pueden volver la cara las Administraciones Públicas exige replantearse el pasado y abordar el futuro desde un contexto diferente, con instrumentos y métodos de trabajo diferentes. Es indudable que en este marco, los recursos humanos, tanto desde una perspectiva económica como puramente organizativa constituye uno de los elementos mediales de mayor relevancia en el seno de lo público. Que dicho proceso se racionalice, tecnifique y reconduzca por pautas que alejen el ámbito e impongan la razón es no sólo una buena noticia para el conjunto de la sociedad, sino esencialmente una obligación permanente de los directivos y servidores públicos que conseguirán con ello hacer más transparente su gestión, sus necesidades y, en último término, el esfuerzo común que justifique su mantenimiento. 
- Director del Gabinete del Secretario de Estado para la Administración Pública.

1 Esta es la tesis mantenida por PAREjo Alfonso en Estado Social y Administración Pública. Los postulados constitucionales de la reforma administrativa. Madrid 1983, pág 95, cuando afirma que "la tópica crisis de la Administración y el Derecho administrativo afecta pues, más bien, como acabamos de ver, al modelo de Estado, en cuanto construcción positiva y dogmática de su organización y funcionamiento; construcción que no se ha revisado al compás de las transformaciones reales, sobrecargando las categorías y las técnicas concebidas y formuladas para soportar otros contenidos..."

2 Especialmente acertado me parece el trabajo de PraTS y Catalí, J. "Derecho y management en las Administraciones Públicas" (notas sobre la crisis y renovación de los respectivos paradigmas) en Revista del CLAD núm. 3, enero de 1995, págs. 91 y ss

${ }^{3}$ GARCÍA DE ENTERRIA, E.: Revolución francesa y Administración contemporánea, $2^{\mathbf{a}}$ ed., p. 27, Madrid, 1981.

${ }^{4}$ Parejo Alfonso L.: Estado..., ob. cit. pág.97.

${ }^{5}$ GarCía de ENTERRía, E.: Ob. cit., p. 65

${ }^{6}$ Sobre la concepción del presupuesto como instrumento de programación y planificación y el alcance del mismo puede verse nuestro libro $E l$ procedimiento administrativo y la ejecución presupuestaria y su control, Madrid 1995.

7 FoRSTHOFF, E.: Tratado de Derecho Administrativo, pp. 584 y ss., Madrid, 1958

${ }^{8} \mathrm{Al}$ tema, en relación con el Estado social se refiere CAmmew, M., en "Las autonomías en la crisis del Estado social", Revista Catalana de Derecho Público, núm. 2-3, diciembre 1985, p. 720

9 FORSTHOFF, E.: Ob. cit.,p. 581

${ }^{10}$ ForsTHOFF, E.: Ob. cit. p. 583

"Sobre el tema puede verse: Posada HeRRera, J. Lecciones de Administración, p. 72, Madrid, 1988.

12 Es curioso comprobar como Estados Unidos en el mismo período pasa del 12 por 100 a, prácticamente, el $35 \%$ que mantiene en los momentos actuales.

13 ARIÑo ORTIZ, G.: Economía y Estado. Crisis y reforma del sector público. Madrid, 1993, pág 27.

${ }^{14}$ ARIÑo ORTIZ, G.: Economía y Estado...Ob. cit., pág 39.

15 El total del capítulo I en los Presupuestos Generales del Estado para 1995 asciende a 4.125 .758 millones de pesetas.
${ }^{16}$ Algunos datos pueden centrar mejor el problema. Así, en diciembre de 1994, el conjunto del personal al servicio de las Administraciones Públicas era de 1.692.529, de los que 610.758 corresponden a la AGE, 40.250 a la Administración de Justicia, 73.528, a las Universidades, 600.961 a las Comunidades Autónomas y 367.032 a las Entidades Locales.

Este proceso contrasta con el de la evolución respectiva, ya que mientras en los últimos catorce años la Administración del Estado ha descendido en 284.973, las Comunidades Autónomas crecen, en el mismo período en $1.251 \%$ efectivo y las Corporaciones Locales en un 119.721. En estos momentos el volumen de las Comunidades Autónomas es prácticamente paritario, en el bien entendido que el personal de éstas últimas procede en 390.832 casos de transferencias y 165.654 son personal de nuevo ingreso en las mismas.

17 Sobre este tema debe verse el ya clásico trabajo de PAREjo ALronso, L.: "La eficacia como principio jurídico de la actuación de la Administración Pública", Documentación Administrativa, núms. 218-219, abril-septiembre de 1989, págs.: 15 a 66; así como el trabajo de OrTeGA AlVAREZ, L.: "El reto dogmático del principio de eficacia". Revista de Administración Pública núm. 133, eneroabril 1994, págs. 7 a 16. Madrid.

${ }^{18}$ Sobre este punto puede verse nuestro trabajo "La incidencia de las nuevas reformas de la Función Pública en la redefinición del Estatuto de los funcionarios públicos". Revista de Administración Pública número 134. Mayo-agosto 1994. Págs. 381 y ss.

${ }^{19}$ En todo caso y teniendo en cuenta el diferente punto de vista del que parten el Derecho laboral y el Derecho Público, parece justificado que entre ambos instrumentos existan claras diferencias, ya que, como se sabe, la Administración Pública parte del principio de la inamovilidad del funcionario, mientras que el Derecho laboral, sin configurar la libertad del empresario en la determinación exacta de sus plantillas sí es verdad que admite diversos sistemas en los que se transmuta la relación jurídica por un equivalente compensatorio. Como quiera que esta perspectiva no se puede deducir de la regulación implícita ni explícita de la Ley 22/1993, es lógico, insistimos, que existan las diferencias entre instrumentos que en modo alguno pueden ser identificados

${ }^{20}$ Aunque no sea éste el momento ni la ocasión sí resulta necesario indicar que una de las medidas que necesariamente deberán acabar de imponerse es la movilidad interorgánica, proceso que se ve dificultado en los momentos actuales por el hecho de entenderse que el principio de personalidad jurídica única no "rige" en el Derecho laboral de las Administraciones Públicas, que se mantienen en un rígido concepto de empresario diferenciado para cada Departamento. A nuestro juicio, los elementos en los que se basa esta rigidez son puramente accidentales y difícilmente compatibles con el principio de personalidad jurídica única de la Administración General del Estado. 


\section{TODOS LOS LIBROS Y PUBLICACIONES} DE LOS DISTINTOS DEPARTAMENTOS MINISTERIALES ESTÁN REUNIDOS EN LA LIBRERIA DEL BOE PARA QUE VD. LOS PUEDA ADQUIRIR SIN DAR MÁS VUELTAS. CÓMODAMENTE. TOME NOTA DE ESTA DIRECCIÓN.

\footnotetext{
TRAFALGAR, 29
}

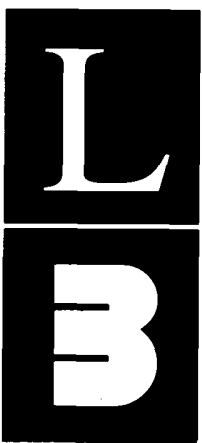

LA

LIBRERIA DEL BOE

28071 MADRID

TELEFONO: 5382295

FAX: 5382267

BASES DE DATOS: 5382222

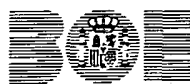

